Commun. Fac. Sci. Univ. Ank. Ser. A1 Math. Stat.

Volume 68, Number 2, Pages 1411-1426(2019)

DOI: $10.31801 /$ cfsuasmas. 455799

ISSN 1303-5991 E-ISSN 2618-6470

http://communications.science.ankara.edu.tr/index.php?series=A1

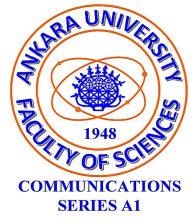

\title{
EQUILIBRIUM AND STABILITY ANALYSIS OF TAKAGI-SUGENO FUZZY DELAYED COHEN-GROSSBERG NEURAL NETWORKS
}

\author{
NEYIR OZCAN
}

\begin{abstract}
This paper carries out an investigation into the problem of the global asymptotic stability of the class of Takagi-Sugeno (T-S) fuzzy delayed Cohen-Grossberg neural networks involving discrete time delays and employing the nondecreasing and slope-bounded activation functions. A new sufficient criterion for the uniqueness and global asymptotic stability of the equilibrium point for this class of fuzzy neural networks is proposed. The uniqueness of the equilibrium point is proved by using the contradiction method, and the stability of the equilibrium point is established by utilizing a novel fuzzy type Lyapunov functional. The obtained stability condition is independent of the time delay parameters and, it can be easily verified by exploiting some commonly used norm properties of matrices. A constructive numerical example is also given to demonstrate the applicability of the proposed stability condition.
\end{abstract}

\section{INTRODUCTION}

Stability and equilibrium properties of Cohen-Grossberg neural network model proposed by Cohen and Grossberg in [1] have been extensively studied due to their potential applications in a variety of fields such as pattern recognition, parallel computation, associative memory design, signal and image processing and optimization. Such types of applications require that the neural network employed for solving these specific problems must possess a unique and globally asymptotically stable equilibrium point. On the other hand, time delays unavoidable exist in the mathematical model of neural networks due to many different reasons. For instance, the finite switching speed of amplifiers in neural systems may cause time delays. The existence of time delays may change the dynamics of the system and cause undesired complex dynamical behaviors. Therefore, it is of crucial importance to consider the effects of time delays when analyzing the stability of neural networks.

Received by the editors: August 29, 2018, Accepted: February 14, 2019.

2010 Mathematics Subject Classification. 15, 34, 93.

Key words and phrases. Stability theory, equilibrium analysis, Cohen-Grossberg neural networks, delayed T-S fuzzy systems.

(C)2019 Ankara University Communications Faculty of Sciences University of Ankara-Series A1 Mathematics and Statistics 
In the recent years, a variety of sufficient conditions for the global asymptotic stability of delayed Cohen-Grossberg neural networks have been proposed [2]-[16].

Fuzzy logic theory has been effectively adopted for modelling the various classes of nonlinear systems to provide a more efficient tool with stability analysis of these systems. In particular, fuzzy systems in the form of the Takagiï-Sugeno (T-S) model [17] have attracted rapidly growing attention in recent years. A T-S fuzzy system is a class of nonlinear systems defined by a set of IF-THEN rules [36]. It has been shown that the T-S model method can provide an effective way with representing complex nonlinear systems by using simple local linear dynamical systems with their linguistic description. Some classes of nonlinear dynamical systems can be approximated by the overall fuzzy linear T-S models for the purpose of stability analysis [18]-[19]. In [20], a sufficient condition for the stability of the T-S fuzzy systems has been proposed by constructing a suitable Lyapunov functional. The methods and techniques used in [19] have been an inspiration for many researchers to extend the T-S fuzzy models to describe different classes of delayed neural networks. Some original and useful results for global stability of various classes of T-S fuzzy delayed neural networks can be found in [21]-[38].

This paper will study the equilibrium and stability properties of the class of T-S fuzzy Cohen-Grossberg neural networks with discrete time delays. First, by using the contradiction method, the condition ensuring the uniqueness of the equilibrium point for this class of neural networks is established. Then, by constructing a suitable fuzzy Lyapunov functional, it will be shown that the condition proposed for the uniqueness of the equilibrium point also implies the global asymptotic stability of the equilibrium point.

\section{System Description and Preliminaries}

Consider the following general Cohen-Grossberg neural network model with discrete time delays:

$$
\dot{x}_{i}(t)=d_{i}\left(x_{i}(t)\right)\left[-c_{i}\left(x_{i}(t)\right)+\sum_{j=1}^{n} a_{i j} f_{j}\left(x_{j}(t)\right)+\sum_{j=1}^{n} b_{i j} f_{j}\left(x_{j}\left(t-\tau_{j}\right)\right)+u_{i}\right],
$$

where $n$ is the number of the neurons in the network, $x_{i}$ denotes the state of the $i$ th neuron, $d_{i}\left(x_{i}\right)$ represents an amplification function, and $c_{i}\left(x_{i}\right)$ is a behaved function. The constants $a_{i j}$ are the neuron interconnection parameters of the neurons within the network, the constants $b_{i j}$ are interconnection parameters of the neurons with time delay parameters $\tau_{j}$. The $f_{i}(\cdot)$ corresponds to the activation functions of neurons. The constants $u_{i}$ are some external inputs. In system (1), $\tau_{j} \geq 0$ represent the time delay parameters with $\tau=\max \left(\tau_{j}\right)$ for $j=1,2, \ldots, n$. The neural system (1) is accompanied by an initial condition of the form: $x_{i}(t)=\phi_{i}(t) \in C([-\tau, 0], R)$, 
where $C([-\tau, 0], R)$ denotes the set of all continuous functions from $[-\tau, 0]$ to $R$.

The usual assumptions on the functions $d_{i}, c_{i}$ and $f_{i}$ are defined to be as follows :

$H_{1}$ : For the amplification functions $d_{i}(x),(i=1,2, \ldots, n)$, there exist positive constants $\psi_{i}$ and $\phi_{i}$ such that $0<\psi_{i} \leq d_{i}(x) \leq \phi_{i}, \forall x \in R$.

$H_{2}$ : For the functions $c_{i}(x),(i=1,2, \ldots, n)$, there exist constants $\gamma_{i}>0$ such that

$$
\frac{c_{i}(x)-c_{i}(y)}{x-y}:=\frac{\left|c_{i}(x)-c_{i}(y)\right|}{|x-y|}: \geq \gamma_{i}>0,: i=1,2, \ldots, n,::: \forall x, y \in R, x \neq y .
$$

$H_{3}$ : For the activation functions $f_{i}(x),(i=1,2, \ldots, n)$, there exist some positive constants $k_{i}$ such that

$$
0 \leq \frac{f_{i}(x)-f_{i}(y)}{x-y} \leq k_{i},:: i=1,2, \cdots, n,:: \forall x, y \in R,: x \neq y .
$$

Now, let $x^{*}$ be an equilibrium point of Cohen-Grossberg neural network model (1). The transformation $z(t)=x(t)-x^{*}$ will shift the equilibrium point $x^{*}$ of system (1) to the origin. The transformed Cohen-Grossberg neural network model is now represented by the following new sets of differential equations :

$$
\dot{z}_{i}(t)=\alpha_{i}\left(z_{i}(t)\right)\left[-\beta_{i}\left(z_{i}(t)\right)+\sum_{j=1}^{n} a_{i j} g_{j}\left(z_{j}(t)\right)+\sum_{j=1}^{n} b_{i j} g_{j}\left(z_{j}\left(t-\tau_{j}\right)\right)\right]
$$

in which the following can be stated

$$
\begin{gathered}
\alpha_{i}\left(z_{i}(t)\right)=d_{i}\left(z_{i}(t)+x_{i}^{*}\right), i=1,2, \ldots, n \\
\beta_{i}\left(z_{i}(t)\right)=c_{i}\left(z_{i}(t)+x_{i}^{*}\right)-c_{i}\left(x_{i}^{*}\right), i=1,2, \ldots, n \\
g_{i}\left(z_{i}(t)\right)=f_{i}\left(z_{i}(t)+x_{i}^{*}\right)-f_{i}\left(x_{i}^{*}\right), i=1,2, \ldots, n .
\end{gathered}
$$

An equivalent mathematical model of (2) can be stated as follows :

$$
\dot{z}(t)=\alpha(z(t))[-\beta(z(t))+A g(z(t))+B g(z(t-\tau))],
$$

where $z(t)=\left(z_{1}(t), z_{2}(t), \ldots, z_{n}(t)\right)^{T}$,

$\alpha(z(t))=\operatorname{diag}\left(\alpha_{1}\left(z_{1}(t)\right), \alpha_{2}\left(z_{2}(t)\right), \ldots, \alpha_{n}\left(z_{n}(t)\right)\right), \quad A=\left(a_{i j}\right)_{n \times n}, B=\left(b_{i j}\right)_{n \times n}$, $\beta(z(t))=\left(\beta_{1}\left(z_{1}(t)\right), \beta_{2}\left(z_{2}(t)\right), \ldots, \beta_{n}\left(z_{n}(t)\right)\right)^{T}$, $g(z(t-\tau))=\left(g_{1}\left(z_{1}\left(t-\tau_{1}\right)\right), g_{2}\left(z_{2}\left(t-\tau_{2}\right)\right), \ldots, g_{n}\left(z_{n}\left(t-\tau_{n}\right)\right)\right)^{T}$.

When introducing the T-S fuzzy model concept into (3), the model of fuzzy Cohen-Grossberg neural network with discrete time delays is obtained as follows [36]:

Plant Rule $r$ : 
IF $\left\{\theta_{1}(t)\right.$ is $\left.M_{r 1}\right\}$ and $\cdots$ and $\left\{\theta_{p}(t)\right.$ is $\left.M_{r p}\right\}$.

\section{THEN}

$$
\dot{z}(t)=\alpha_{r}(z(t))\left[-\beta_{r}(z(t))+A_{r} g(z(t))+B_{r} g(z(t-\tau))\right],
$$

where $\theta_{l}(t)(l=1,2, \ldots, p)$ are the premise variables. $M_{r l}(r \in\{1,2, \ldots, m\}, \quad l \in$ $\{1,2, \ldots, p\}$ are the fuzzy sets and $m$ is the number of IF-THEN rules.

By inferring from the fuzzy models, the final model of a fuzzy Cohen-Grossberg neural network takes the following form [36] :

$$
\dot{z}(t)=\sum_{r=1}^{m} h_{r}(\theta(t))\left\{\alpha_{r}(z(t))\left[-\beta_{r}(z(t))+A_{r} g(z(t))+B_{r} g(z(t-\tau))\right]\right\},
$$

where $\theta(t)=\left[\theta_{1}(t), \theta_{2}(t), \ldots, \theta_{p}(t)\right]^{T}, \omega_{r}(\theta(t))=\prod_{l=1}^{p} M_{r l}\left(\theta_{l}(t)\right)$ and $h_{r}(\theta(t))=$ $\frac{\omega_{r}(\theta(t))}{\sum_{r=1}^{m} \omega_{r}(\theta(t))}$ denote the weight and averaged weight of each fuzzy rule, respectively. The term $M_{r l}\left(\theta_{l}(t)\right)$ is the grade membership of $\theta_{l}(t)$ in $M_{r l}$. We assume that $\omega_{r}(\theta(t)) \geq 0, \quad r \in\{1,2, \ldots, m\}$. Therefore, we have $\sum_{r=1}^{m} h_{r}(\theta(t))=1$ for all $t \geq 0$.

Note that $A_{r}=\left(a_{i j}^{(r)}\right)_{n \times n}, B_{r}=\left(b_{i j}^{(r)}\right)_{n \times n}$, $\alpha_{r}(z(t))=\operatorname{diag}\left(\alpha_{r 1}\left(z_{1}(t)\right), \alpha_{r 2}\left(z_{2}(t)\right), \ldots, \alpha_{r n}\left(z_{n}(t)\right)\right)$, $\beta_{r}(z(t))=\left(\beta_{r 1}\left(z_{1}(t)\right), \beta_{r 2}\left(z_{2}(t)\right), \ldots, \beta_{r n}\left(z_{n}(t)\right)\right)^{T}, r=1,2, \ldots, m$.

We also note that, in system (5), the assumptions $H_{1}, H_{2}$ and $H_{3}$ can now be respectively adopted as follows :

$$
\begin{aligned}
& A_{1}: 0<\psi_{r i} \leq \alpha_{r i}\left(z_{i}(t)\right) \leq \phi_{r i}, \quad i=1,2, \ldots, n, \quad r=1,2, \ldots, m \\
& A_{2}: z_{i}(t) \beta_{r i}\left(z_{i}(t)\right) \geq \gamma_{r i} z_{i}^{2}(t) \geq 0, \quad i=1,2, \ldots, n, \quad r=1,2, \ldots, m \\
& A_{3}:\left|g_{i}\left(z_{i}(t)\right)\right| \leq k_{i}\left|z_{i}(t)\right|, \quad z_{i}(t) g_{i}\left(z_{i}(t)\right) \geq 0, \quad i=1,2, \ldots, n .
\end{aligned}
$$

\section{Main Result}

In this section, we will present two main theorems. The first theorem proves the uniqueness of equilibrium point for system (5), which is stated as follows :

Theorem 1. Under the assumptions $A_{1}, A_{2}$ and $A_{3}$, the origin $z=0$ of the T-S fuzzy Cohen-Grossberg neural network model defined by (5) is the unique equilibrium point if there exist positive constants $\xi_{r}, r=1,2, \cdots, m$ such that the following condition holds :

$$
\Omega=2 \psi \gamma K^{-1}-\sum_{r=1}^{m} \phi_{r}\left(\left|A_{r}\right|+\left|A_{r}^{T}\right|\right)-\sum_{r=1}^{m} \frac{1}{\xi_{r}} \phi_{r}^{2}|| B_{r} \|_{2}^{2} I-\sum_{r=1}^{m} \xi_{r} I>0,
$$


where $\psi=\min \left\{\psi_{r}\right\}$ with $\psi_{r}=\min \left\{\psi_{r i}\right\}, \gamma=\min \left\{\gamma_{r}\right\}$ with $\gamma_{r}=\min \left\{\gamma_{r i}\right\}$, $\phi_{r}=\max \left\{\phi_{r i}\right\}, i=1,2, \cdots, n, r=1,2, \cdots, m, K=\operatorname{diag}\left(k_{1}, k_{2}, \cdots, k_{n}\right)$ and $\left|A_{r}\right|=\left(\left|a_{i j}^{(r)}\right|\right)_{n \times n}$.

Proof. We will prove this theorem by using the contradiction method. Let $z \neq 0$ be an equilibrium point of system (5). Then, we have

$$
\dot{z}(t)=\sum_{r=1}^{m} h_{r}(\theta(t))\left\{\alpha_{r}(z)\left[-\beta_{r}(z)+A_{r} g(z)+B_{r} g(z)\right]\right\}=0
$$

which can be written as

$$
-\sum_{r=1}^{m} h_{r}(\theta(t)) \alpha_{r}(z) \beta_{r}(z)+\sum_{r=1}^{m} h_{r}(\theta(t)) \alpha_{r}(z) A_{r} g(z)+\sum_{r=1}^{m} h_{r}(\theta(t)) \alpha_{r}(z) B_{r} g(z)=0 .
$$

Let $z \neq 0$ and $g(z)=0$. Then, one has

$$
\sum_{r=1}^{m} h_{r}(\theta(t)) \alpha_{r}(z) \beta_{r}(z)=0
$$

and from (8)

$$
\sum_{r=1}^{m} h_{r}(\theta(t)) z^{T} \alpha_{r}(z) \beta_{r}(z)=0
$$

Thus, we obtain

$$
\sum_{r=1}^{m} h_{r}(\theta(t)) z^{T} \alpha_{r}(z) \beta_{r}(z) \geq \psi \gamma z^{T} z>0, \forall z \neq 0 .
$$

It is clear that if $z \neq 0$, then (7) cannot be satisfied. Therefore, at the equilibrium point, when $g(z)=0, z \neq 0$ cannot be a solution of (6).

Let $z \neq 0$ and $g(z) \neq 0$. Then, we can write

$$
\begin{aligned}
& -2 \sum_{r=1}^{m} h_{r}(\theta(t)) g^{T}(z) \alpha_{r}(z) \beta_{r}(z)+2 \sum_{r=1}^{m} h_{r}(\theta(t)) g^{T}(z) \alpha_{r}(z) A_{r} g(z) \\
& +2 \sum_{r=1}^{m} h_{r}(\theta(t)) g^{T}(z) \alpha_{r}(z) B_{r} g(z)=0 .
\end{aligned}
$$

We note that

$$
\begin{aligned}
-2 \sum_{r=1}^{m} h_{r}(\theta(t)) g^{T}(z) \alpha_{r}(z) \beta_{r}(z) & =-2 \sum_{r=1}^{m} h_{r}(\theta(t)) \sum_{i=1}^{n} \alpha_{r i}\left(z_{i}\right) \beta_{r i}\left(z_{i}\right) g_{i}\left(z_{i}\right) \\
& \leq-2 \sum_{r=1}^{m} h_{r}(\theta) \sum_{i=1}^{n} \psi_{r i} \gamma_{r i} z_{i} g_{i}\left(z_{i}\right) \\
& \leq-2 \sum_{r=1}^{m} h_{r}(\theta(t)) \sum_{i=1}^{n} \frac{1}{k_{i}} \psi_{r i} \gamma_{r i} g_{i}^{2}\left(z_{i}\right)
\end{aligned}
$$




$$
\begin{aligned}
\leq & -2 \sum_{r=1}^{m} h_{r}(\theta(t)) g^{T}(z) \psi \gamma K^{-1} g(z) \\
= & -2 \psi \gamma\left|g^{T}(z(t))\right| K^{-1}|g(z(t))|, \\
\sum_{r=1}^{m} h_{r}(\theta(t))\left\{2 g^{T}(z) \alpha_{r}(z) A_{r} g(z)\right\} & \leq \sum_{r=1}^{m} 2\left|g^{T}(z)\right| \alpha_{r}(z)\left|A_{r} \| g(z)\right| \\
& \leq \sum_{r=1}^{m} 2\left|g^{T}(z)\right| \phi_{r}\left|A_{r} \| g(z)\right| \\
& =\sum_{r=1}^{m} \phi_{r}\left|g^{T}(z)\right|\left(\left|A_{r}\right|+\left|A_{r}^{T}\right|\right)|g(z)|, \quad(3) \\
\sum_{r=1}^{m} h_{r}(\theta(t)) 2 g^{T}(z) \alpha_{r}(z) B_{r} g(z) \leq & \sum_{r=1}^{m} h_{r}(\theta(t)) 2\left\|\alpha_{r}(z)\right\|_{2}\left\|B_{r}\right\|_{2}\|g(z)\|_{2}\|g(z)\|_{2} \\
\leq & \sum_{r=1}^{m} 2 \phi_{r}\left\|B_{r}\right\|_{2}|| g(z)\left\|_{2}\right\| g(z) \|_{2} \\
\leq & \sum_{r=1}^{m} \frac{1}{\xi_{r}} \phi_{r}^{2}\left\|B_{r}\right\|_{2}^{2}\|g(z)\|_{2}^{2}+\sum_{r=1}^{m} \xi_{r}\|g(z)\|_{2}^{2} . \quad(4)
\end{aligned}
$$

Using (11)-(13) in (10) yields

$$
\begin{aligned}
& -2 \psi \gamma\left|g^{T}(z)\right| K^{-1}|g(z)|+\sum_{r=1}^{m} \phi_{r}\left|g^{T}(z)\right|\left(\left|A_{r}\right|+\left|A_{r}^{T}\right|\right)|g(z)| \\
& +\sum_{r=1}^{m} \frac{1}{\xi_{r}} \phi_{r}^{2}\left\|B_{r}\right\|_{2}^{2}\|g(z)\|_{2}^{2}+\sum_{r=1}^{m} \xi_{r}\|g(z)\|_{2}^{2} \geq 0
\end{aligned}
$$

which is of the form

$$
\left|g^{T}(z)\right|(-\Omega)|g(z)| \geq 0
$$

or equivalently

$$
\left|g^{T}(z)\right| \Omega|g(z)| \leq 0 .
$$

On the other hand, if $\Omega$ is a positive definite matrix, then, for all $g(z(t)) \neq 0$, we have

$$
\left|g^{T}(z)\right| \Omega|g(z)|>0 .
$$

Obviously, when $\Omega>0$, (14) contradicts with (15), implying that under the condition of Theorem 1, the equilibrium equation of system (5) given by (6) cannot have a solution where $g(z) \neq 0$. Thus, we can conclude that Theorem 1 guarantees that the origin of system (5) is the unique equilibrium point.

We will now present the following theorem that proves the stability of system $(5)$. 
Theorem 2. Under the assumptions $A_{1}, A_{2}$ and $A_{3}$, the T-S fuzzy Cohen-Grossberg neural network model defined by (5) is globally asymptotically stable if there exist positive constants $\xi_{r}, r=1,2, \cdots, m$ such that the following condition holds :

$$
\Omega=2 \psi \gamma K^{-1}-\sum_{r=1}^{m} \phi_{r}\left(\left|A_{r}\right|+\left|A_{r}^{T}\right|\right)-\sum_{r=1}^{m} \frac{1}{\xi_{r}} \phi_{r}^{2}\left\|B_{r}\right\|_{2}^{2} I-\sum_{r=1}^{m} \xi_{r} I>0,
$$

where $\psi=\min \left\{\psi_{r}\right\}$ with $\psi_{r}=\min \left\{\psi_{r i}\right\}, \gamma=\min \left\{\gamma_{r}\right\}$ with $\gamma_{r}=\min \left\{\gamma_{r i}\right\}$, $\phi_{r}=\max \left\{\phi_{r i}\right\}, i=1,2, \cdots, n, r=1,2, \cdots, m, K=\operatorname{diag}\left(k_{1}, k_{2}, \cdots, k_{n}\right)$ and $\left|A_{r}\right|=\left(\left|a_{i j}^{(r)}\right|\right)_{n \times n}$.

Proof. Consider the following positive definite Lyapunov functional :

$$
\begin{aligned}
V(z(t))= & z^{T}(t) z(t)+2 \varepsilon \sum_{i=1}^{n} \int_{0}^{z_{i}(t)} g_{i}(s) d s \\
& +\varepsilon \sum_{r=1}^{m} \xi_{r} \sum_{j=1}^{n} \int_{t-\tau_{j}}^{t} g_{j}^{2}\left(z_{j}(\zeta)\right) d \zeta+\eta \sum_{j=1}^{n} \int_{t-\tau_{j}}^{t} g_{j}^{2}\left(z_{j}(\zeta)\right) d \zeta,
\end{aligned}
$$

where $\varepsilon$ and $\eta$ are some positive constants to be determined later. We can calculate the time derivative of $V(z(t))$ along the trajectories of neural system (5) as follows

$$
\begin{aligned}
\dot{V}(z(t))= & 2 z^{T}(t) \dot{z}(t)+2 \varepsilon \sum_{i=1}^{n} g_{i}\left(z_{i}(t)\right) \dot{z}_{i}(t)=2 z^{T}(t) \dot{z}(t)+2 \varepsilon g^{T}(z(t)) \dot{z}(t) \\
& +\varepsilon \sum_{r=1}^{m} \xi_{r} \sum_{j=1}^{n} g_{j}^{2}\left(z_{j}(t)\right)-\varepsilon \sum_{r=1}^{m} \xi_{r} \sum_{j=1}^{n} g_{j}^{2}\left(z_{j}\left(t-\tau_{j}\right)\right) \\
& +\eta \sum_{j=1}^{n} g_{j}^{2}\left(z_{j}(t)\right)-\eta \sum_{j=1}^{n} g_{j}^{2}\left(z_{j}\left(t-\tau_{j}\right)\right) \\
= & 2 z^{T}(t) \sum_{r=1}^{m} h_{r}(\theta(t))\left\{\alpha_{r}(z(t))\left[-\beta_{r}(z(t))+A_{r} g(z(t))+B_{r} g(z(t-\tau))\right]\right\} \\
& +2 \varepsilon g^{T}(z(t)) \sum_{r=1}^{m} h_{r}(\theta(t))\left\{\alpha_{r}(z(t))\left[-\beta_{r}(z(t))+A_{r} g(z(t))+B_{r} g(z(t-\tau))\right]\right\} \\
& +\varepsilon \sum_{r=1}^{m} \xi_{r}\|g(z(t))\|_{2}^{2}-\varepsilon \sum_{r=1}^{m} \xi_{r}\|g(z(t-\tau))\|_{2}^{2} \\
& +\eta\|g(z(t))\|_{2}^{2}-\eta\|g(z(t-\tau))\|_{2}^{2} \\
= & \sum_{r=1}^{m} h_{r}(\theta(t))\left\{-2 z^{T}(t) \alpha_{r}(z(t)) \beta_{r}(z(t))\right\}
\end{aligned}
$$




$$
\begin{aligned}
& +\sum_{r=1}^{m} h_{r}(\theta(t))\left\{2 z^{T}(t) \alpha_{r}(z(t)) A_{r} g(z(t))\right\} \\
& +\sum_{r=1}^{m} h_{r}(\theta(t))\left\{2 z^{T}(t) \alpha_{r}(z(t)) B_{r} g(z(t-\tau))\right\} \\
& +\varepsilon \sum_{r=1}^{m} h_{r}(\theta(t))\left\{-2 g^{T}(z(t)) \alpha_{r}(z(t)) \beta_{r}(z(t))\right\} \\
& +\varepsilon \sum_{r=1}^{m} h_{r}(\theta(t))\left\{2 g^{T}(z(t)) \alpha_{r}(z(t)) A_{r} g(z(t))\right\} \\
& +\varepsilon \sum_{r=1}^{m} h_{r}(\theta(t))\left\{2 g^{T}(z(t)) \alpha_{r}(z(t)) B_{r} g(z(t-\tau))\right\} \\
& +\varepsilon \sum_{r=1}^{m} \xi_{r}\|g(z(t))\|_{2}^{2}-\varepsilon \sum_{r=1}^{m} \xi_{r}\|g(z(t-\tau))\|_{2}^{2} \\
& +\eta\|g(z(t))\|_{2}^{2}-\eta\|g(z(t-\tau))\|_{2}^{2} .
\end{aligned}
$$

We first note the following inequalities :

$$
\begin{aligned}
\sum_{r=1}^{m} h_{r}(\theta(t))\left\{-2 z^{T}(t) \alpha_{r}(z(t)) \beta_{r}(z(t))\right\} & =\sum_{r=1}^{m} h_{r}(\theta(t))\left\{-2 \sum_{i=1}^{n} \alpha_{r i}\left(z_{i}(t)\right) \beta_{r i}\left(z_{i}(t)\right) z_{i}(t)\right\} \\
& \leq \sum_{r=1}^{m} h_{r}(\theta(t))\left\{-2 \sum_{i=1}^{n} \psi_{r i} \gamma_{r i} z_{i}^{2}(t)\right\} \\
& \leq \sum_{r=1}^{m} h_{r}(\theta(t))\left\{-2 \psi_{r} \gamma_{r} \sum_{i=1}^{n} z_{i}^{2}(t)\right\} \\
& \leq-2 \psi \gamma \sum_{r=1}^{m} h_{r}(\theta(t))\left\{\sum_{i=1}^{n} z_{i}^{2}(t)\right\} \\
& =-2 \psi \gamma\|z(t)\|_{2}^{2} \sum_{r=1}^{m} h_{r}(\theta(t)) \\
& =-2 \psi \gamma\|z(t)\|_{2}^{2}, \\
\sum_{r=1}^{m} h_{r}(\theta(t))\left\{2 z^{T}(t) \alpha_{r}(z(t)) A_{r} g(z(t))\right\} & \\
\sum_{r=1}^{m} h_{r}(\theta(t))\left\{-\left(\sqrt{\psi \gamma} z(t)-\frac{1}{\sqrt{\psi \gamma}}\right.\right. & \left.\alpha_{r}(z(t)) A_{r} g(z(t))\right)^{T}(\sqrt{\psi \gamma} z(t)
\end{aligned}
$$




$$
\begin{aligned}
& \left.\left.-\frac{1}{\sqrt{\psi \gamma}} \alpha_{r}(z(t)) A_{r} g(z(t))\right)\right\}+\sum_{r=1}^{m} h_{r}(\theta(t))\left\{\psi \gamma z^{T}(t) z(t)\right. \\
& \left.\left.+\frac{1}{\psi \gamma} g^{T}(z(t)) A_{r}^{T} \alpha_{r}^{2}(z(t)) A_{r} g(z(t))\right)\right\} \\
& \left.\leq \sum_{r=1}^{m} h_{r}(\theta(t))\left\{\leq \psi \gamma z^{T}(t) z(t)+\frac{1}{\psi \gamma} g^{T}(z(t)) A_{r}^{T} \alpha_{r}^{2}(z(t)) A_{r} g(z(t))\right)\right\} \\
& \leq \sum_{r=1}^{m} h_{r}(\theta(t)) \psi \gamma z^{T}(t) z(t)+\sum_{r=1}^{m} h_{r}(\theta(t)) \frac{1}{\psi \gamma}\left\|\alpha_{r}^{2}(z(t))\right\|_{2}\left\|A_{r}\right\|_{2}^{2}\|g(z(t))\|_{2}^{2} \\
& \leq \sum_{r=1}^{m} h_{r}(\theta(t)) \psi \gamma\|z(t)\|_{2}^{2}+\sum_{r=1}^{m} h_{r}(\theta(t)) \frac{\phi^{2}}{\psi \gamma}\left\|A_{r}\right\|_{2}^{2}\|g(z(t))\|_{2}^{2} \\
& \leq \psi \gamma\|z(t)\|_{2}^{2}+\sum_{r=1}^{m} \frac{\phi^{2}}{\psi \gamma}\left\|A_{r}\right\|_{2}^{2}\|g(z(t))\|_{2}^{2}, \\
& \sum_{r=1}^{m} h_{r}(\theta(t))\left\{2 z^{T}(t) \alpha_{r}(z(t)) B_{r} g(z(t-\tau))\right\} \\
& =\sum_{r=1}^{m} h_{r}(\theta(t))\left\{-\left(\sqrt{\psi \gamma} z(t)-\frac{1}{\sqrt{\psi \gamma}} \alpha_{r}(z(t)) B_{r} g(z(t-\tau))\right)^{T}\right. \\
& \left.\times\left(\sqrt{\psi \gamma} z(t)-\frac{1}{\sqrt{\psi \gamma}} \alpha_{r}(z(t)) B_{r} g(z(t-\tau))\right)\right\} \\
& \left.+\sum_{r=1}^{m} h_{r}(\theta(t))\left\{\psi \gamma z^{T}(t) z(t)+\frac{1}{\psi \gamma} g^{T}(z(t-\tau)) B_{r}^{T} \alpha_{r}^{2}(z(t)) B_{r} g(z(t-\tau))\right)\right\} \\
& \left.\leq \sum_{r=1}^{m} h_{r}(\theta(t))\left\{\leq \psi \gamma z^{T}(t) z(t)+\frac{1}{\psi \gamma} g^{T}(z(t-\tau)) B_{r}^{T} \alpha_{r}^{2}(z(t)) B_{r} g(z(t-\tau))\right)\right\} \\
& \leq \sum_{r=1}^{m} h_{r}(\theta(t)) \psi \gamma z^{T}(t) z(t)+\sum_{r=1}^{m} h_{r}(\theta(t)) \frac{1}{\psi \gamma}\left\|\alpha_{r}^{2}(z(t))\right\|_{2}\left\|B_{r}\right\|_{2}^{2}\|g(z(t-\tau))\|_{2}^{2} \\
& \leq \sum_{r=1}^{m} h_{r}(\theta(t)) \psi \gamma\|z(t)\|_{2}^{2}+\sum_{r=1}^{m} h_{r}(\theta(t)) \frac{\phi^{2}}{\psi \gamma}\left\|B_{r}\right\|_{2}^{2}\|g(z(t-\tau))\|_{2}^{2} \\
& \leq \psi \gamma\|z(t)\|_{2}^{2}+\sum_{r=1}^{m} \frac{\phi^{2}}{\psi \gamma}\left\|B_{r}\right\|_{2}^{2}\|g(z(t-\tau))\|_{2}^{2}, \\
& \varepsilon \sum_{r=1}^{m} h_{r}(\theta(t))\left\{-2 g^{T}(z(t)) \alpha_{r}(z(t)) \beta_{r}(z(t))\right\}
\end{aligned}
$$




$$
\begin{aligned}
& =\varepsilon \sum_{r=1}^{m} h_{r}(\theta(t))\left\{-2 \sum_{i=1}^{n} \alpha_{r i}\left(z_{i}(t)\right) \beta_{r i}\left(z_{i}(t)\right) g_{i}\left(z_{i}(t)\right)\right\} \\
& \leq \varepsilon \sum_{r=1}^{m} h_{r}(\theta(t))\left\{-2 \sum_{i=1}^{n} \psi_{r i} \gamma_{r i} z_{i}(t) g_{i}\left(z_{i}(t)\right)\right\} \\
& \leq \varepsilon \sum_{r=1}^{m} h_{r}(\theta(t))\left\{-2 \sum_{i=1}^{n} \frac{1}{k_{i}} \psi_{r i} \gamma_{r i} g_{i}^{2}\left(z_{i}(t)\right)\right\} \\
& \leq \varepsilon \sum_{r=1}^{m} h_{r}(\theta(t))\left\{-2 g^{T}(z(t)) \psi \gamma K^{-1} g(z(t))\right\} \\
& \leq-2 \varepsilon \psi \gamma\left|g^{T}(z(t))\right| K^{-1}|g(z(t))|, \\
& \varepsilon \sum_{r=1}^{m} h_{r}(\theta(t))\left\{2 g^{T}(z(t)) \alpha_{r}(z(t)) A_{r} g(z(t))\right\} \\
& \leq \varepsilon \sum_{r=1}^{m} 2\left|g^{T}(z(t))\right| \alpha_{r}(z(t))\left|A_{r} \| g(z(t))\right| \\
& \leq \varepsilon \sum_{r=1}^{m} 2\left|g^{T}(z(t))\right| \phi_{r}\left|A_{r}\right||g(z(t))| \\
& =\varepsilon \sum_{r=1}^{m} \phi_{r}\left|g^{T}(z(t))\right|\left(\left|A_{r}\right|+\left|A_{r}^{T}\right|\right)|g(z(t))|, \\
& \varepsilon \sum_{r=1}^{m} h_{r}(\theta(t))\left\{-2 g^{T}(z(t)) \alpha_{r}(z(t)) B_{r} g(z(t-\tau))\right\} \\
& \varepsilon \leq \sum_{r=1}^{m} h_{r}(\theta(t))\left\{2\left\|\alpha_{r}(z(t))\right\|_{2}\left\|B_{r}\right\|_{2}\|g(z(t))\|_{2}\|g(z(t-\tau))\|_{2}\right\} \\
& \varepsilon \leq \sum_{r=1}^{m} 2 \phi_{r}\left\|B_{r}\right\|_{2}\|g(z(t))\|_{2}\|g(z(t-\tau))\|_{2} \\
& \leq \varepsilon \sum_{r=1}^{m} \frac{1}{\xi_{r}} \phi_{r}^{2}\left\|B_{r}\right\|_{2}^{2}\|g(z(t))\|_{2}^{2}+\varepsilon \sum_{r=1}^{m} \xi_{r}\|g(z(t-\tau))\|_{2}^{2} .
\end{aligned}
$$

Using (17)-(22) in (16) leads to

$$
\begin{aligned}
\dot{V}(z(t)) \leq & -2 \psi \gamma\|z(t)\|_{2}^{2}+\psi \gamma\|z(t)\|_{2}^{2}+\sum_{r=1}^{m} \frac{\phi^{2}}{\psi \gamma}\left\|A_{r}\right\|_{2}^{2}\|g(z(t))\|_{2}^{2} \\
& +\psi \gamma\|z(t)\|_{2}^{2}+\sum_{r=1}^{m} \frac{\phi^{2}}{\psi \gamma}\left\|B_{r}\right\|_{2}^{2}\|g(z(t-\tau))\|_{2}^{2}
\end{aligned}
$$




$$
\begin{aligned}
& -2 \varepsilon \psi \gamma\left|g^{T}(z(t))\right| K^{-1}|g(z(t))| \\
& +\varepsilon \sum_{r=1}^{m}\left|g^{T}(z(t))\right|\left(\phi_{r}\left|A_{r}\right|+\left|A_{r}^{T}\right| \phi_{r}\right)|g(z(t))| \\
& +\varepsilon \sum_{r=1}^{m} \frac{1}{\xi_{r}} \phi_{r}^{2}\left\|B_{r}\right\|_{2}^{2}|| g(z(t))\left\|_{2}^{2}+\varepsilon \sum_{r=1}^{m} \xi_{r}|| g(z(t-\tau))\right\|_{2}^{2} \\
& +\varepsilon \sum_{r=1}^{m} \xi_{r}|| g(z(t))\left\|_{2}^{2}-\varepsilon \sum_{r=1}^{m} \xi_{r}\right\| g(z(t-\tau)) \|_{2}^{2} \\
& +\eta\|g(z(t))\|_{2}^{2}-\eta\|g(z(t-\tau))\|_{2}^{2} .
\end{aligned}
$$

Let $\|A\|_{2}=\max \left\{\left\|A_{r}\right\|_{2}\right\}$ and $\|B\|_{2}=\max \left\{\left\|B_{r}\right\|_{2}\right\}, r=1,2, \cdots, m$ and $\eta=\frac{m \phi^{2}}{\psi \gamma}$. Then, (23) takes the form

$$
\begin{aligned}
\dot{V}(z(t)) \leq & \frac{m \phi^{2}}{\psi \gamma}\|A\|_{2}^{2}|| g(z(t))\left\|_{2}^{2}+\frac{m \phi^{2}}{\psi \gamma}\right\| B\left\|_{2}^{2}\right\| g(z(t)) \|_{2}^{2} \\
& -2 \varepsilon \psi \gamma\left|g^{T}(z(t))\right| K^{-1}|g(z(t))|+\varepsilon \sum_{r=1}^{m}\left|\phi_{r} g^{T}(z(t))\right|\left(\left|A_{r}\right|+\left|A_{r}^{T}\right|\right)|g(z(t))| \\
& +\varepsilon \sum_{r=1}^{m} \frac{1}{\xi_{r}} \phi_{r}^{2}\left\|B_{r}\right\|_{2}^{2}\|g(z(t))\|_{2}^{2}+\varepsilon \sum_{r=1}^{m} \xi_{r}\|g(z(t))\|_{2}^{2} \\
= & \frac{m \phi^{2}}{\psi \gamma}\left(\|A\|_{2}^{2}+\|B\|_{2}^{2}\right)\|g(z(t))\|_{2}^{2} \\
& -\varepsilon\left|g^{T}(z(t))\right|\left(2 \psi \gamma K^{-1}-\sum_{r=1}^{m}\left(\phi_{r}\left(\left|A_{r}\right|+\left|A_{r}^{T}\right|\right)\right.\right. \\
& \left.\left.+\frac{1}{\xi_{r}} \phi_{r}^{2}\left\|B_{r}\right\|_{2}^{2} I+\xi_{r} I\right)\right)|g(z(t))| \\
= & \frac{m \phi^{2}}{\psi \gamma}\left(\|A\|_{2}^{2}+\|B\|_{2}^{2}\right)\|g(z(t))\|_{2}^{2}-\varepsilon\left|g^{T}(z(t))\right| \Omega|g(z(t))| \\
\leq & \frac{m \phi^{2}}{\psi \gamma}\left(\|A\|_{2}^{2}+\|B\|_{2}^{2}\right)\|g(z(t))\|_{2}^{2}-\varepsilon \lambda_{m}(\Omega)\|g(z(t))\|_{2}^{2}
\end{aligned}
$$

where $\lambda_{m}(\Omega)>0$ is the minimum eigenvalue of the positive definite matrix $\Omega$. The choice

$$
\varepsilon>\frac{m \phi^{2}}{\psi \gamma \lambda_{m}(\Omega)}\left(\|A\|_{2}^{2}+\|B\|_{2}^{2}\right)
$$

ensures that $\dot{V}(z(t))$ expressed by $(24)$ is negative definite for all $g(z(t)) \neq 0$, or equivalently $\dot{V}(z(t))<0$ for all $z(t) \neq 0$ as $g(z(t)) \neq 0$ implies that $z(t) \neq 0$. In the case where $z(t)=0,(z(t)=0$ implies that $g(z(t))=0), \dot{V}(z(t))$ directly takes 
the form

$$
\begin{aligned}
\dot{V}(z(t)) & =-\varepsilon \sum_{r=1}^{m} \xi_{r} \sum_{j=1}^{n} g_{j}^{2}\left(z_{j}\left(t-\tau_{j}\right)\right)-\eta \sum_{j=1}^{n} g_{j}^{2}\left(z_{j}\left(t-\tau_{j}\right)\right) \\
& \leq-\eta \sum_{j=1}^{n} g_{j}^{2}\left(z_{j}\left(t-\tau_{j}\right)\right)=-\eta g^{T}(z(t-\tau)) g(z(t-\tau)) .
\end{aligned}
$$

It follows from $(25)$ that if $g(z(t-\tau)) \neq 0$, then $\dot{V}(z(t))<0$. Note that $\dot{V}(z(t))=0$ if and only if $z(t)=g(z(t))=g(z(t-\tau))=0$. On the other hand, one can easily check that $V(z(t))$ is radially unbounded. Therefore, from the standard Lyapunov stability theorems, we can conclude that the condition given in Theorem 2 establishes the global asymptotic stability of the origin of neural system (5).

Remark 3. In [21]-[38], the stability of fuzzy neural system (5) have been established by using the LMI (linear matrix inequality approach) or the M-matrix based approach. It should be pointed out here that stability conditions expressed in the LMI forms need to be checked for negative definiteness of the high dimensional matrices formed by network parameters of neural systems. On the other hand, the M-matrixbased approach neglects the sign of entries of the interconnection matrices, which may result in the possible conservativeness in the stability criteria. However, the stability conditions proposed in Theorems 1 and 2 establish a simple and easily verifiable relationship between the network parameters of the system without using the LMI-based or M-matrix-based approaches. Therefore, the result proposed in the current paper can be considered as an alternative condition to the previously published results.

Now, the following numerical example is given in order to demonstrate the applicability of the theoretical results obtained in Theorems 1 and 2.

Example 4. Let Takagi-Sugeno fuzzy delayed neural network (5) be defined by the parameters $\psi=1, \gamma=1, \phi_{1}=\phi_{2}=\phi_{3}=\phi_{4}=1, k_{1}=k_{2}=k_{3}=k_{4}=1$ and by the system matrices :

$$
\begin{aligned}
A_{1} & =\left[\begin{array}{cccc}
a & a & a & a \\
a & a & a & a \\
-a & -a & -a & -a \\
-a & a & a & -a
\end{array}\right], A_{2}=\left[\begin{array}{cccc}
-a & -a & -a & -a \\
a & -a & -a & a \\
a & -a & -a & a \\
a & a & a & a \\
& & &
\end{array}\right], \\
A_{3}= & {\left[\begin{array}{cccc}
-a & -a & a & a \\
-a & -a & a & a \\
-a & a & a & -a \\
-a & -a & -a & a
\end{array}\right], A_{4}=\left[\begin{array}{cccc}
a & -a & -a & -a \\
-a & a & -a & -a \\
a & -a & a & -a \\
a & a & -a & -a
\end{array}\right], }
\end{aligned}
$$




$$
\begin{aligned}
B_{1}= & {\left[\begin{array}{cccc}
b & b & b & b \\
b & b & -b & -b \\
b & -b & b & -b \\
-b & b & b & -b
\end{array}\right], B_{2}=\left[\begin{array}{cccc}
-b & -b & -b & -b \\
-b & -b & b & b \\
-b & b & -b & b \\
b & -b & -b & b
\end{array}\right], } \\
B_{3} & =\left[\begin{array}{cccc}
-b & -b & -b & -b \\
b & b & -b & -b \\
b & -b & b & -b \\
-b & b & b & -b
\end{array}\right], B_{4}=\left[\begin{array}{cccc}
b & b & b & b \\
-b & -b & b & b \\
-b & b & -b & b \\
b & -b & -b & b
\end{array}\right],
\end{aligned}
$$

where $a>0$ and $b>0$ are some positive real constants. From the above matrices, we obtain

$$
\left|A_{1}\right|+\left|A_{1}^{T}\right|=\left|A_{2}\right|+\left|A_{2}^{T}\right|=\left|A_{3}\right|+\left|A_{3}^{T}\right|=\left|A_{4}\right|+\left|A_{4}^{T}\right|=2\left[\begin{array}{cccc}
a & a & a & a \\
a & a & a & a \\
a & a & a & a \\
a & a & a & a \\
& & &
\end{array}\right]
$$

and

$$
\left\|B_{1}\right\|_{2}=\left\|B_{2}\right\|_{2}=\left\|B_{3}\right\|_{2}=\left\|B_{4}\right\|_{2}=2 b .
$$

Let $\xi_{1}=\xi_{2}=\xi_{3}=\xi_{4}=2 b$. Then, $\Omega$ in Theorem 2 is obtained as follows :

$$
\begin{aligned}
\Omega & =\left[\begin{array}{llll}
2 & 0 & 0 & 0 \\
0 & 2 & 0 & 0 \\
0 & 0 & 2 & 0 \\
0 & 0 & 0 & 2
\end{array}\right]-\left[\begin{array}{cccc}
8 a & 8 a & 8 a & 8 a \\
8 a & 8 a & 8 a & 8 a \\
8 a & 8 a & 8 a & 8 a \\
8 a & 8 a & 8 a & 8 a \\
& &
\end{array}\right]-\left[\begin{array}{cccc}
8 b & 0 & 0 & 0 \\
0 & 8 b & 0 & 0 \\
0 & 0 & 8 b & 0 \\
0 & 0 & 0 & 8 b \\
& &
\end{array}\right] \\
& =2\left[\begin{array}{cccc}
1-4 a-4 b & -4 a & -4 a & -4 a \\
-4 a & 1-4 a-4 b & -4 a & -4 a \\
-4 a & -4 a & 1-4 a-4 b & -4 a \\
-4 a & -4 a & -4 a & 1-4 a-4 b
\end{array}\right],
\end{aligned}
$$

where $\Omega>0$ if $1-16 a-4 b>0$. Thus, for the network parameters of this example, the stability condition for system (5) is derived to be $16 a+4 b<1$.

\section{Conclusion}

This paper has presented a sufficient condition for the uniqueness and global asymptotic stability of the equilibrium point for the class of Takagi-Sugeno (T-S) fuzzy delayed Cohen-Grossberg neural networks with discrete time delays in the presence of the nondecreasing and slope-bounded activation functions. The uniqueness of the equilibrium point has been proved by using the contradiction method, and the stability of the equilibrium point has been established by employing a new fuzzy 
type Lyapunov functional. A numerical example has been presented to support the effectiveness of the proposed stability criterion. The advantage of the obtained condition over the previously published literature results has also been addressed.

\section{REFERENCES}

[1] Cohen, M.A. and Grossberg, S., Absolute stability and global pattern formation and parallel memory storage by competitive neural networks, IEEE Transactions on Systems, Man and Cybernetics, vol. 13, (1983), 815-821.

[2] Li, L. and Jian, J., Exponential convergence and Lagrange stability for impulsive CohenGrossberg neural networks with time-varyin delays, Journal of Computational and Applied Mathematics, vol. 277, (2015), 23-35.

[3] Yu, S,. Zhang, Z. and Quan, Z., New global exponential stability conditions for inertial CohenGrossberg neural networks with time delays, Neurocomputing, vol. 151, (2015), 1446-1454.

[4] Liu, Y., Liu, W., Obaid, M. A. and Abbas, I. A., Exponential stability of Markovian jumping Cohen-Grossberg neural networks with mixed mode-dependent time-delays, Neurocomputing, vol. 177, (2016), 409-415.

[5] Esteves, S. and Oliveira, J. J., Global asymptotic stability of nonautonomous CohenGrossberg neural network models with infinite delays, Applied Mathematics and Computation, vol. 265, (2015), 333-346.

[6] Ozcan, N., New conditions for global stability of neutral-type delayed Cohen-Grossberg neural networks, Neural Networks, vol. 106, (2018), 1-7.

[7] Du, Y. and Xu, R., Multistability and multiperiodicity for a class of Cohen-Grossberg BAM neural networks with discontinuous activation functions and time delays, Neural Processing Letters, vol. 42, (2015), 417-435.

[8] Kao, Y., Wang, C. and Zhang, L., Delay-dependent robust exponential stability of impulsive Markovian jumping reaction-diffusion Cohen-Grossberg neural networks, Neural Processing Letters, vol. 38, (2013), 321-346.

[9] Li, R., Cao, J., Alsaedi, A. and Ahmad, B., Passivity analysis of delayed reaction-diffusion Cohen-Grossberg neural networks via Hardy-Poincare inequality, Journal of the Franklin Institute, vol. 354, (2017), 3021-3038.

[10] Li, B. and Song, Q., Some new results on periodic solution of Cohen-Grossberg neural network with impulses, Neurocomputing, vol. 177, (2016), 401-408.

[11] Nie, X., Zheng, W. X. and Cao, J., Multistability of memristive Cohen-Grossberg neural networks with non-monotonic piecewise linear activation functions and time-varying delays, Neural Networks, vol. 71, (2015), 27-36.

[12] Wei, T., Wang, L. and Wang, Y., Existence, uniqueness and stability of mild solutions to stochastic reaction-diffusion Cohen-Grossberg neural networks with delays and Wiener processes, Neurocomputing, vol. 239, (2017), 19-27.

[13] Zheng, C.D., Shan, Q. H., Zhang, H. and Wang, Z., On stabilization of stochastic CohenGrossberg neural networks with mode-dependent mixed time-delays and Markovian switching, IEEE Transactions on Neural Networks and Learning Systems, vol. 24, (2013), 800-811.

[14] Xu, C. and Zhang, Q., On antiperiodic solutions for Cohen-Grossberg shunting inhibitory neural networks with time-varying delays and impulses, Neural Computation, vol. 26, (2014), $2328-2349$.

[15] Zhu, Q.X. and Cao, J.D., Robust exponential stability of Markovian jump impulsive stochastic Cohen-Grossberg neural networks with mixed time delays, IEEE Transactions on Neural Networks and Learning Systems, vol. 21, (2010), 1314-1325.

[16] Arik, S. and Orman, Z., Global stability analysis of Cohen-Grossberg neural networks with time varying delays, Physics Letters A, vol. 341, (2005), 410-421. 
[17] Takagi, T. and Sugeno, M., Fuzzy identification of systems and its applications to modeling and control, IEEE Transactions on Systems, Man and Cybernetics, vol. 15, (1983), 116-132.

[18] Hou, Y.Y., Liao, T.L. and Yan, J.J., Stability analysis of Takagi-Sugeno fuzzy cellular neural networks with time-varying delays, IEEE Transactions on Systems, Man and Cybernetics, vol. 37, (2007), 720-726.

[19] Yamamoto, H. and Furuhashi, T., A new sufficient condition for stable fuzzy control system and its design method, IEEE Transactions on Fuzzy Systems, vol. 9, (2001), 554-569.

[20] Huang, H., Ho, D.W.C. and Lam, J., Stochastic stability analysis of fuzzy Hopfield neural networks with time-varying delays, IEEE Transactions on Circuits Systems-I, Fundamental Theory and Applications, vol. 52, (2005), 251-255.

[21] Bao, H., Existence and exponential stability of periodic solution for BAM fuzzy CohenGrossberg neural networks with mixed delays, Neural Processing Letters, vol. 43, (2016), $871-885$.

[22] Zheng, C. D., Zhang, X. and Wang, Z., Mode and delay-dependent stochastic stability conditions of fuzzy neural networks with Markovian jump parameters, Neural Processing Letters, vol. 43, (2016), 195-217.

[23] Yang, W., Periodic solution for fuzzy Cohen-Grossberg BAM neural networks with both timevarying and distributed delays and variable coefficients, Neural Processing Letters, vol. 40, (2014), 51-73.

[24] Gan, Q., Exponential synchronization of stochastic fuzzy cellular neural networks with reaction-diffusion terms via periodically intermittent control, Neural Processing Letters, vol. 37, (2013), 393-410.

[25] Chandran, R. and Balasubramaniam, P., Delay dependent exponential stability for fuzzy recurrent neural networks with interval time-varying delay, Neural Processing Letters, vol. 37, (2013), 147-161.

[26] Tseng, K.H, Tsai, J. S. and Lu, C. Y., Design of delay-dependent exponential estimator for T-S Fuzzy Neural networks with mixed time-varying interval delays using hybrid TaguchiGenetic algorithm, Neural Processing Letters, vol. 36, (2012), 49-67.

[27] Ahn, C. K., Takagi-Sugeno fuzzy Hopfield neural networks for H-infinity nonlinear system identification, Neural Processing Letters, vol. 34, (2011), 59-70.

[28] Gan, Q., Xu, R. and Yang, P., Stability analysis of stochastic fuzzy cellular neural networks with time-varying delays and reaction-diffusion terms, Neural Processing Letters, vol. 32,( 2010), 45-57.

[29] Yang, W., Yu, W., Cao, J., Alsaadi, F. E. and Hayat, T., Global exponential stability and lag synchronization for delayed memristive fuzzy Cohen-Grossberg BAM neural networks with impulses, Neural Networks, vol. 98, (2018), 122-153.

[30] Jian, J. and Jiang, W., Lagrange exponential stability for fuzzy Cohen-Grossberg neural networks with time-varying delays, Fuzzy Sets and Systems, vol. 277, (2017), 65-80.

[31] Muralisankar, S. and Gopalakrishnan, N., Robust stability criteria for Takagi-Sugeno fuzzy Cohen-Grossberg neural networks of neutral type, Neurocomputing, vol. 144, (2014), 516-525.

[32] Li, C., Li, Y. and Ye, Y., Exponential stability of fuzzy Cohen-Grossberg neural networks with time delays and impulsive effects, Communications in Nonlinear Science and Numerical Simulation, vol. 15, (2010), 3599-3606.

[33] Zhu, Q. and Li, X., Exponential and almost sure exponential stability of stochastic fuzzy delayed Cohen-Grossberg neural networks, Fuzzy Sets and Systems, vol. 203, (2012), 74-94.

[34] Mathiyalagan, K., Park, J H., Sakthivel, R. and Anthoni, S. M., Delay fractioning approach to robust exponential stability of fuzzy Cohen-Grossberg neural networks, Applied Mathematics and Computation, vol.230, (2014), 451-463.

[35] Bao, G., Wen, S. and Zeng, Z., Robust stability analysis of interval fuzzy Cohen-Grossberg neural networks with piecewise constant argument of generalized type, Neural Networks, vol. $33,2012,32-41$. 
[36] Balasubramaniam, P. and Ali, M. S., Stability analysis of Takagi-Sugeno fuzzy CohenGrossberg BAM neural networks with discrete and distributed time-varying delays, Mathematical and Computer Modelling, vol. 53, (2011), 151-160.

[37] Li, C., Li, Y. and Ye, Yuan, Exponential stability of fuzzy Cohen-Grossberg neural networks with time delays and impulsive effects, Communications in Nonlinear Science and Numerical Simulation, vol. 15, (2010), pp. 3599-3606.

[38] $\mathrm{He}, \mathrm{D}$. and $\mathrm{Xu}, \mathrm{D} .$, Attracting and invariant sets of fuzzy Cohen-Grossberg neural networks with time-varying delays, Physics Letters A, vol. 372, (2008), 7057-7062.

Current address: Neyir Ozcan: Department of Electrical-Electronics Engineering Faculty of Engineering, Bursa Uludag University Bursa, Turkey

E-mail address: neyir@uludag.edu.tr

ORCID Address: http://orcid.org/0000-0002-5513-9072 